\title{
Active RFID TAGs System Analysis of Energy Consumption As Excitable Linear Bifurcation System
}

\author{
Ofer Aluf \\ Department of Physics, Ben-Gurion University of the Negev, Be'er-Sheva,
}

Israel

\section{Introduction}

In this article, Very Critical and useful subject is discussed: Active RFID TAGs system energy analysis as excitable linear bifurcation system. Active RFID TAGs have a built in power supply, such as a battery, as well as electronics that perform specialized tasks. By contrast, passive RFID TAGs do not have a power supply and must rely on the power emitted by a RFID Reader to transmit data. Thus, if a reader is not present, the passive TAGs cant communicate an data. Active TAGs can communicate in the absence of a reader. Active RFID TAGs system energy consumption can be function of many variables : $q(m), u(m)$, $z(m), t(m)$, tms $(m)$, when $m$ is the number of TAG IDs which are uniformly distributed in the interval $[0,1)$. It is very important to emphasis that basic Active RFID TAG, equivalent circuit is Capacitor (Cic), Resistor (Ric), L (RFID's Coil inductance as a function of overall Coil's parameters) all in parallel and Voltage generator $\mathrm{Vs}(\mathrm{t})$ with serial parasitic resistance. The Voltage generator and serial parasitic resistance are in parallel to all other Active RFID TAG's elements (Cic, Ric, and L (Coil inductance)). The Active RFID TAG equivalent circuit can be represent as a differential equation which depending on variable parameters. The investigation of Active RFID's differential equation based on bifurcation theory, the study of possible changes in the structure of the orbits of a differential equation depending on variable parameters. The article first illustrate certain observations and analyze local bifurcations of an appropriate arbitrary scalar differential equation. Finally investigate Active RFID TAGs system energy for the best performance using excitable bifurcation diagram.

\section{Energy aware anti collision protocol for active RFID TAGs system}

Active RFID TAGs have a built in power supply, such as a battery. The major advantages of an active RFID TAGs are: It can be read at distances of one hundred feet or more, greatly improving the utility of the device. It may have other sensors that can use electricity for power. The disadvantages of an active RFID TAGs are: The TAG cannot function without battery power, which limits the lifetime of the TAG. The TAG is typically more expensive. The TAG is physically larger, which may limit applications. The long term maintenance costs for an active RFID tag can be greater than those of a passive TAGs if the batteries are

Source: Radio Frequency Identification Fundamentals and Applications, Design Methods and Solutions, Book edited by: Cristina Turcu, ISBN 978-953-7619-72-5, pp. 324, February 2010, INTECH, Croatia, downloaded from SCIYO.COM 
replaced. Battery outages in an active TAGs can result in expensive misreads. Active RFID TAGs may have all or some of the following features: Longest communication range of any TAG. The capability to perform independent monitoring and control.

The capability of initiating communications. The capabilities of performing diagnostics. The highest data bandwidth. The active RFID TAGs may even be equipped with autonomous networking; the TAGs autonomously determine the best communication path. Mainly active RFID TAGs have a built in power supply, such as battery, as well as electronics that perform specialized tasks. By By contrast, passive RFID TAGs do not have a power supply and must rely on the power emitted by a RFID Reader to transmit data. There is an arbitration while reading TAGs (TAGs anti collision problem). First identify and then read data stored in RFID TAGs.

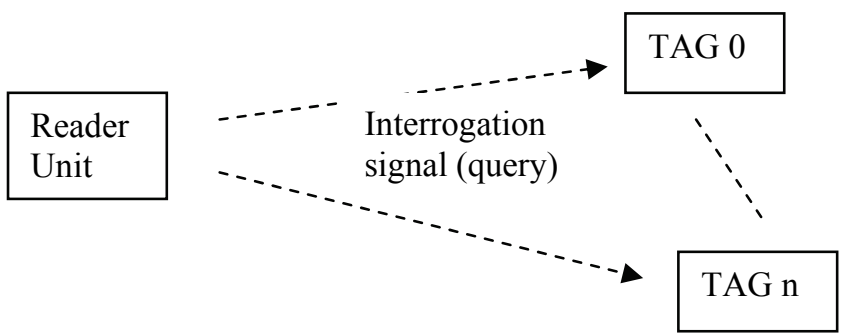

Fig. 1.

It is very important to read TAG IDs of all. The Anti collision protocol based on two methods: ALOHA and its variants and Binary tree search. ALOHA protocol reducing collisions by separating TAG responds by time (probabilistic and simple). TAG ID may not be read for a very long time. The Binary tree search protocol is deterministic in nature. Read all TAGs by successively querying nodes at a different levels of the tree with TAG IDs distributed on the tree based on there prefix. Guarantee that all TAGs IDs will be read within a certain time frame. The binary tree search procedure, however, uses up a lot of reader queries and TAG responses by relying on colliding responses of TAGs to determine which sub tree to query next. Higher energy consumption at readers and TAGs (If they are active TAGs). TAGs cant be assumed to be able to communicate with each other directly. TAGs may not be able of storing states of the arbitration process in their memory. There are three anti collision protocols: Alls include and combine ideas of a binary tree search protocol with frame slotted ALOHA, deterministic schemes, and energy aware. The first anti collision protocol is Multi Slotted (MS) scheme, multiple slots per query to reduce the chances of collision among the TAG responses. The second anti collision protocol is Multi Slotted with Selective sleep (MSS) scheme, using sleep commands to put resolved TAGs to sleep during the arbitration process. Both MS and MSS have a probabilistic flavor, TAGs choose a reply slot in a query frame randomly. The third anti collision protocol is Multi Slotted with Assigned slots (MAS), assigning TAGs in each sub tree of the search tree to a specific slot of the query frame. It's a deterministic protocol, including the replay behavior of TAGs. All three protocols can adjusting the frame size used per query. Maximize energy savings at the reader by reducing collisions among TAG responses. The frame size is also chosen based on a specified average time constraint within which all TAGs IDs must be read. The binary search protocols are Binary Tree (BT) and Query Tree (QT). Both work by splitting TAG IDs using queries from the reader until all TAGs are read. 
Binary Tree (BT) relies on TAGs remembering results of previous inquiries by the readers. TAGs susceptible to their power supply. Query Tree (QT) protocol, is a deterministic TAG anti collision protocol, which is memory less with TAGs requiring no additional memory except that required to store their ID.

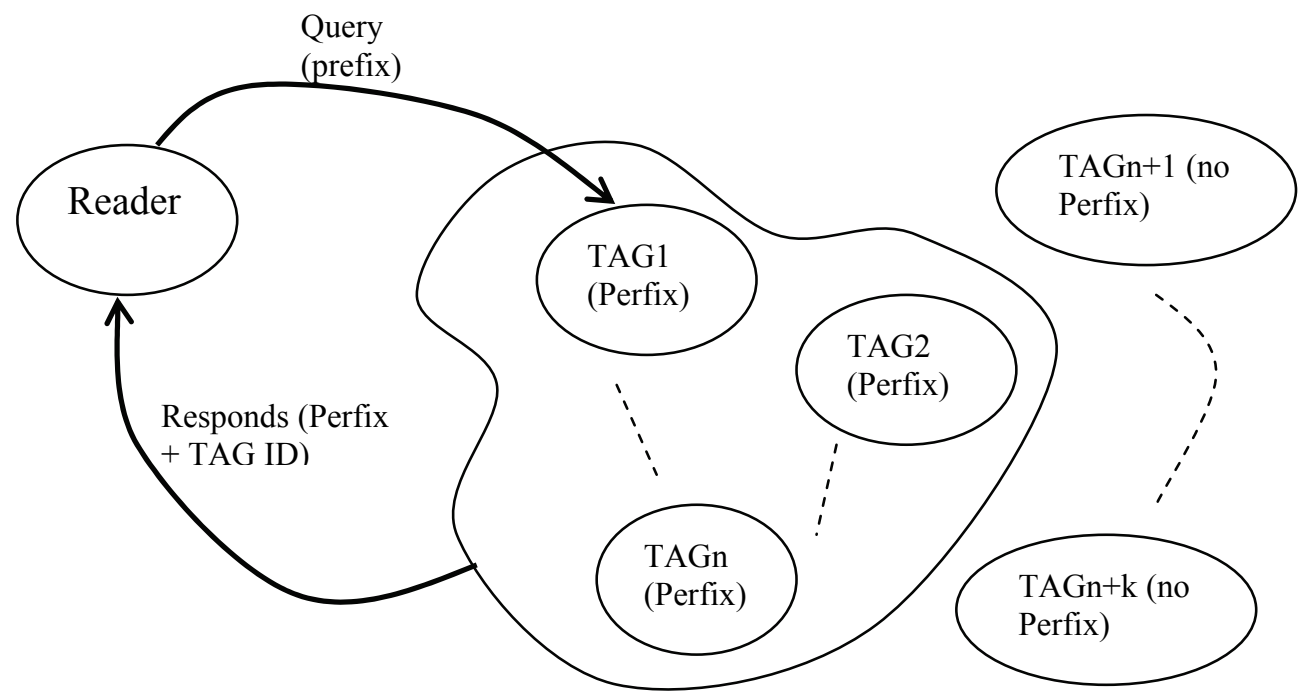

Fig. 2.

The approach to energy aware anti collision protocols for RFID systems is to combine the deterministic nature of binary search algorithms along with the simplicity of frame slotted ALOHA to reduce the number of TAG response collisions. The QT protocol relies on colliding responses to queries that are sent to internal modes of a tree to determine the location of TAG ID. Allow TAGs to transmit responses within a slotted time frame and thus, try to avoid collisions with responses from other TAGs. The energy consumption at the reader is a function of the number of queries it sends, and number of slots spent in the receive mode. Energy consumption at an active TAG is function of the number of queries received by the TAG and the number of responses it sends back. Neglect the energy spent in modes other than transmit and receive for simplicity. Assumption: Time slot in which a reader query or message is sent is equal to the duration as that of a TAG response. The

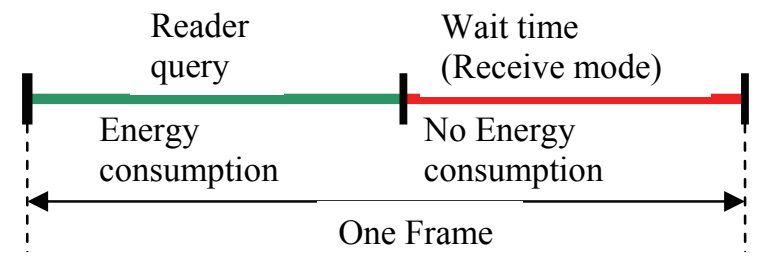

Start of

End of

Frame

Frame

Fig. 3. 
energy model of the reader is based upon a half duplex operation. Reader transmits energy and its query for a specific period and then waits in receive mode with no more energy transmission until end of frame. The flow chart for reader query and TAGs response mechanism is as below:

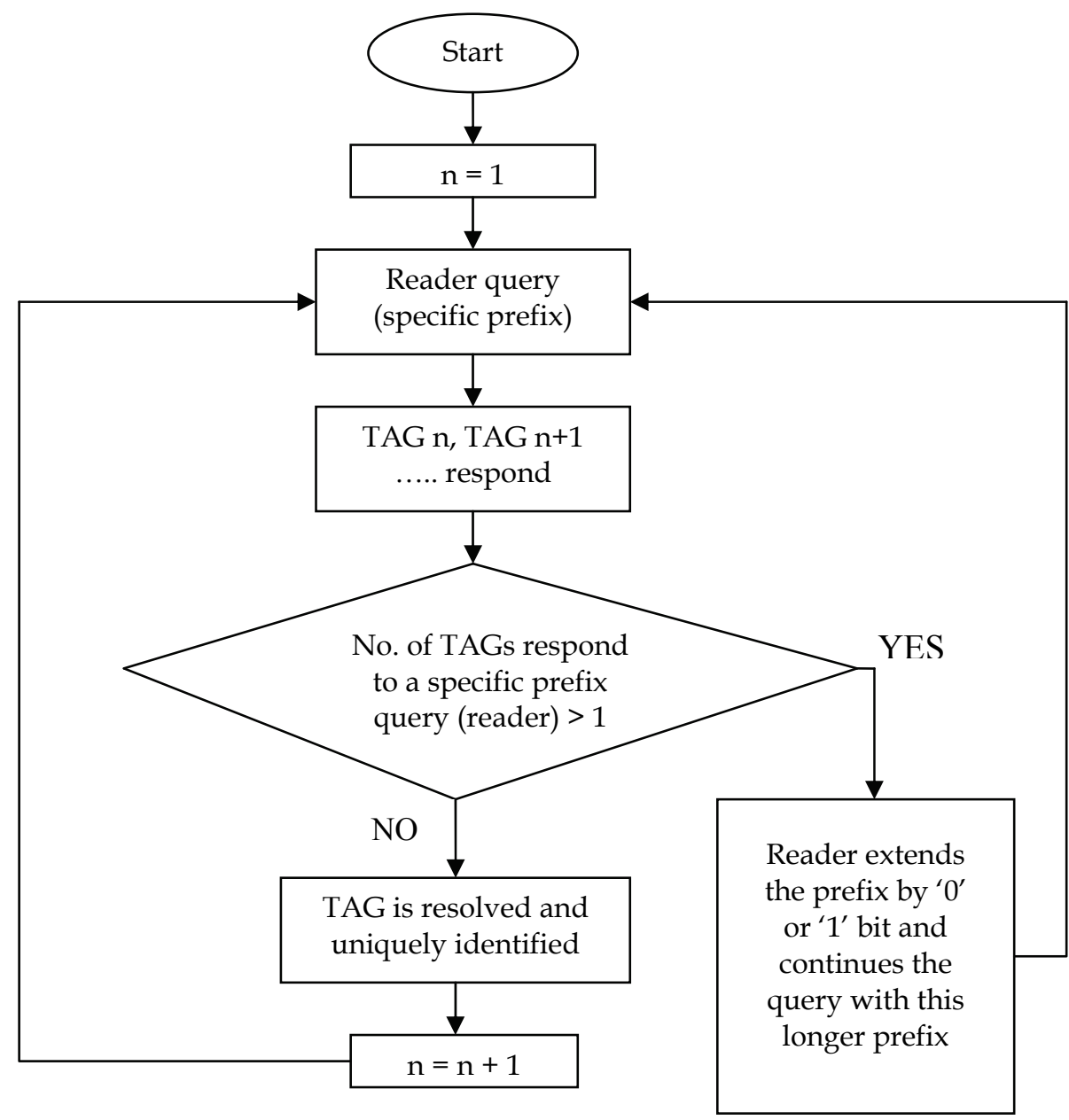

Fig. 4.

Pulse based half duplex operation is termed as sequential (SEQ) operation.

\begin{tabular}{|c|c|}
\hline $\begin{array}{c}\text { Power required by the reader } \\
\text { to transmit }\end{array}$ & $\begin{array}{c}\text { Power required by the reader } \\
\text { to receive }\end{array}$ \\
\hline PRtx & PRrx \\
\hline
\end{tabular}

Table 1.

And 


\begin{tabular}{|c|c|}
\hline $\begin{array}{c}\text { Power required by an active } \\
\text { TAG to transmit }\end{array}$ & $\begin{array}{c}\text { Power required by an active } \\
\text { TAG to receive }\end{array}$ \\
\hline PTtx & PTrx \\
\hline
\end{tabular}

Table 2.

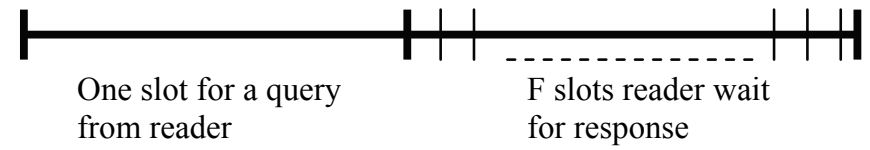

Fig. 5.

Reader energy consumption: $\mathrm{q}(\mathrm{m})^{*}\left(\mathrm{PRtx}+\mathrm{PRrx}{ }^{*} \mathrm{~F}\right)$ when $\mathrm{q}(\mathrm{m})$ is the number of queries for read $m$ TAGs. The energy consumption of all active TAGs: $q(m)^{*}$ PTrx $+u(m) * P T t x$ when $\mathrm{q}(\mathrm{m})$ is the number of reader queires, $\mathrm{u}(\mathrm{m})$ is the number of TAG responses. For MSS scheme (include sleep command) the reader energy consumption is

$$
\mathrm{q}(\mathrm{m}) \text { * }(\mathrm{PRtx}+\mathrm{PRrx} * \mathrm{~F})+\mathrm{z}(\mathrm{m}) \text { * PRtx. }
$$

The total energy consumption for all active TAGs is

$$
\mathrm{q}(\mathrm{m}) * \mathrm{PTr} x+\mathrm{u}(\mathrm{m}) * \mathrm{PTt} \mathrm{x}+\mathrm{z}(\mathrm{m}) \text { * PTrx, }
$$

when $z(m)$ is the number of sleep commands issued by the reader. The average analysis of energy consumption:

$$
\begin{aligned}
& q(m)--- \text { average..number..of..reader..queires. } \\
& - \\
& u^{(m)} \text { - average number of TAG responses. } \\
& - \\
& z^{(m)} \text { - average number of sleep commands } \\
& \text { issued by the reader (only for MSS Scheme) } \\
& - \\
& t^{(m)} \text { - average number of time slots required } \\
& \text { to read all TAGs. } \\
& - \\
& t_{M S}(m) \text { - average number of time slots required to read } m \text { TAGs }
\end{aligned}
$$

$\mathrm{m}$ TAG IDs are uniformly distributed in the interval [0.1].

I get the expression for One active RFID TAG total energy consumption:

$$
\text { TAG Power }=\frac{1}{m} *\left[q(m) \cdot P_{T r x}+U(m) \cdot P_{T t x}+Z(m) \cdot P_{T r x}\right]
$$

\section{Active RFID TAG equivalent circuit}

Active RFID TAG can be represent as a parallel Equivalent Circuit of Capacitor and Resistor in parallel with Supply voltage source (internal resistance). 


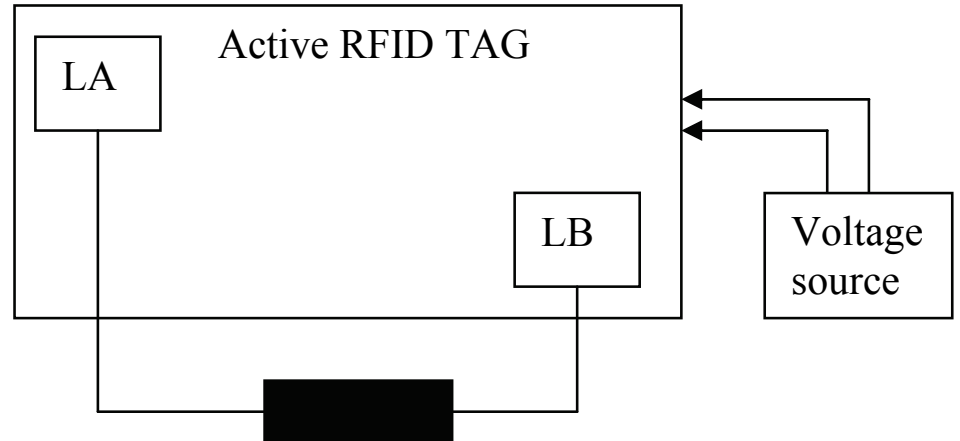

\section{Antenna}

Fig. 6.

The Active RFID TAG Antenna can be represents as Parallel inductor to the basic Active RFID Equivalent Circuit. The simplified complete equivalent circuit of the label is as below:

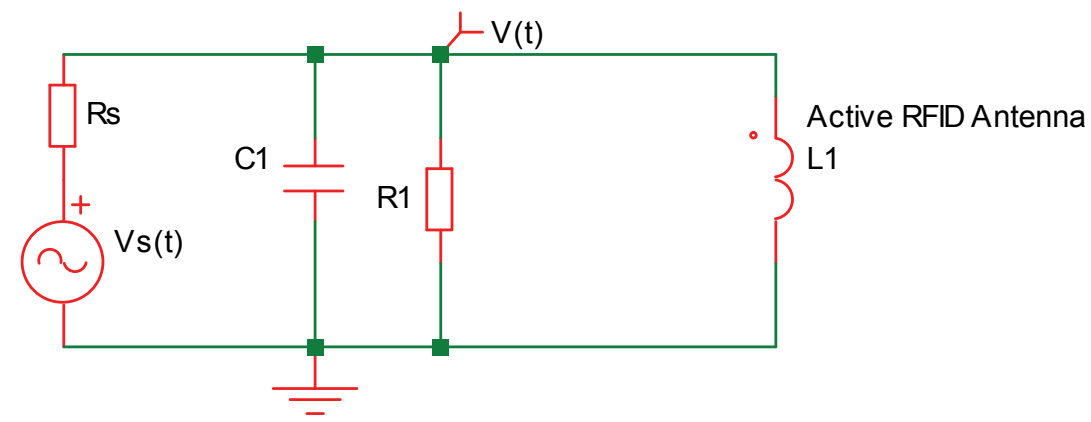

\section{Active RFID's Equivalent circuit}

Fig. 7.

$$
\begin{aligned}
& V_{L 1}=L 1 \cdot \frac{d I}{d t} \Rightarrow I_{L 1}=\frac{1}{L 1} \cdot \int_{0}^{t 1} V_{L 1} \cdot d t, \quad I_{C 1}=C 1 \cdot \frac{d V c 1}{d t}, \quad \sum_{j=1}^{4} I j=0 \\
& V=V c 1=V_{L 1}=V_{R 1} \\
& \frac{V}{R 1}+C 1 \cdot \frac{d V}{d t}+\frac{1}{L 1} \cdot \int_{0}^{t 1} V \cdot d t+\frac{V-V s(t)}{R s}=0 \\
& \left\{\frac{1}{R 1} \cdot \frac{d V}{d t}+C 1 \cdot \frac{d^{2} V}{d t^{2}}+\frac{1}{L 1} \cdot V+\frac{d V}{d t} \cdot \frac{1}{R s}\right\} \stackrel{\frac{d V_{s}(t)}{d t} \rightarrow \varepsilon(0<\varepsilon<<1)}{\longrightarrow} 0 \\
& \varepsilon>1 \Rightarrow \ddot{V} \cdot C 1+\left(\frac{1}{R 1}+\frac{1}{R s}\right) \cdot \dot{V}+\frac{1}{L 1} \cdot V=\frac{1}{R s} \cdot \dot{V}_{S}(\mathrm{t}) \\
& \frac{1}{R 1} \cdot \frac{d V}{d t}+C 1 \cdot \frac{d^{2} V}{d t^{2}}+\frac{1}{L 1} \cdot V+\left[\frac{d V}{d t}-\frac{d V s(t)}{d t}\right] \cdot \frac{1}{R s}=0
\end{aligned}
$$




$$
\begin{aligned}
& V 2=\frac{d V 1}{d t}=\frac{d V}{d t}, \mathrm{~V} 1=\mathrm{V} \\
& \frac{d V 1}{d t}=V 2, \frac{d V 2}{d t}=-\left[\frac{1}{C 1 \cdot R 1}+\frac{1}{R s \cdot C 1}\right] \cdot V 2-\frac{1}{C 1 \cdot L 1} \cdot V 1+\frac{1}{R s \cdot C 1} \cdot \frac{d V s(t)}{d t} \\
& \left(\begin{array}{l}
\frac{d V 1}{d t} \\
\frac{d V 2}{d t}
\end{array}\right)=\left(\begin{array}{cc}
0 & 1 \\
-\frac{1}{C 1 \cdot L 1} & -\left[\frac{1}{C 1 \cdot R 1}+\frac{1}{R s \cdot C 1}\right]
\end{array}\right) \cdot\left(\begin{array}{l}
V 1 \\
V 2
\end{array}\right)+\left(\begin{array}{l}
0 \\
\frac{1}{R s \cdot C 1} \cdot \frac{d V s(t)}{d t}
\end{array}\right) \\
& \text { Lcalc }=\left[\frac{\mu 0}{\pi} *[X 1+X 2-X 3+X 4]^{*} N c^{p}\right]
\end{aligned}
$$

, $\mathrm{L} 1=$ Lcalc

$$
\begin{aligned}
& X 1=\operatorname{Aavg}^{*} \ln \left(\frac{2 * A a v g * B a v g}{d^{*}\left(A a v g+\sqrt{A a v g^{2}+B a v g^{2}}\right)}\right) \\
& X 2=B a v g * \ln \left(\frac{2 * A a v g * B a v g}{d^{*}\left(B a v g+\sqrt{A a v g^{2}+B a v g^{2}}\right)}\right) \\
& X 3=2 *\left[A a v g+B a v g-\sqrt{\left[A a v g^{2}+B a v g^{2}\right]}\right]
\end{aligned}
$$

$X 4=(A a v g+B a v g) / 4$, The RFID's coil calculation inductance expression is

Definition of limits, Estimations: Track thickness $t, \mathrm{Al}$ and $\mathrm{Cu}$ coils ( $\mathrm{t}>30 \mathrm{um})$. The printed coils as high as possible. Estimation of turn exponent $\mathrm{p}$ is needed for inductance calculation.

\begin{tabular}{|c|c|}
\hline Coil manufacturing technology & P \\
\hline Wired & $1.8-1.9$ \\
\hline Etched & $1.75-1.85$ \\
\hline Printed & $1.7-1.8$ \\
\hline
\end{tabular}

Table 3.

Active RFID can be considered as Van der Pol's system. Van der Pol's equation provides an example of an oscillator with nonlinear damping, energy being dissipated at large amplitudes and generated at low amplitudes. Such systems typically posses limit cycles, sustained oscillations a round a state at which energy generation and dissipation balance. The basic Van der Pol's equation can be written in the form:

$$
\begin{gathered}
\ddot{X}+\alpha \cdot \phi(x) \cdot \dot{X}+X=\beta \cdot \rho(t) \\
\varepsilon>1 \Rightarrow \ddot{V} \cdot C 1+\left(\frac{1}{R 1}+\frac{1}{R s}\right) \cdot \dot{V}+\frac{1}{L 1} \cdot V=\frac{1}{R s} \cdot \dot{V}_{S}(t)
\end{gathered}
$$




$$
\begin{aligned}
\varepsilon>>1 \Rightarrow & \ddot{V}+\frac{1}{\mathrm{C} 1} \cdot\left(\frac{1}{R 1}+\frac{1}{R s}\right) \cdot \dot{V}+\frac{1}{\mathrm{~L} 1 \cdot \mathrm{C} 1} \cdot \mathrm{V}=\frac{1}{R s \cdot \mathrm{C} 1} \cdot \dot{V}_{S}(\mathrm{t}) \\
& X \rightarrow V, \quad \alpha \cdot \phi(x) \rightarrow \frac{1}{\mathrm{C} 1} \cdot\left(\frac{1}{R 1}+\frac{1}{R s}\right) \\
& \frac{1}{\mathrm{~L} 1 \cdot \mathrm{C} 1} \rightarrow 1, \frac{1}{R s \cdot C 1} \cdot \dot{V}_{S}(\mathrm{t}) \rightarrow \beta \cdot \rho(t)
\end{aligned}
$$

Lets define:

$$
\begin{aligned}
& f_{S}(\mathrm{t})=\dot{V}_{S}(\mathrm{t}) \text { then } \triangleleft \\
& \varepsilon>>1 \Rightarrow \ddot{V} \cdot C 1+\left(\frac{1}{R 1}+\frac{1}{R S}\right) \cdot \dot{V}+\frac{1}{L 1} \cdot V=\frac{1}{R S} \cdot f_{S}(\mathrm{t})
\end{aligned}
$$

then " $\mathrm{f}$ " is a " $\mathrm{T}$ " periodic function of the independent variable $\mathrm{t}$, and $\lambda=\frac{1}{R s}$

The term $\lambda \cdot f_{S}(\mathrm{t})=\frac{1}{R s} \cdot \dot{V}_{S}(\mathrm{t})$ is called the forcing function.

$\lambda \rightarrow 0 \Rightarrow \frac{1}{R s} \rightarrow 0 \Rightarrow R s \rightarrow \infty$ there is no forcing and the system act as Van Der Pol Oscillator.

It is necessary to examine the trajectories $(\mathrm{V} 1, \mathrm{~V} 2, \mathrm{t})$ of the non-autonomous Active RFID system in $\mathbb{R}^{2} x \mathbb{R}$ rather than the orbits in $\mathbb{R}^{2}$. Equivalently, we may consider the orbits of the Active RFID TAGs three dimensional autonomous system.

$$
\begin{aligned}
& \frac{d V 1}{d t}=V 2 \\
& \frac{d V 2}{d t}=-\left[\frac{1}{C 1 \cdot R 1}+\frac{1}{R s \cdot C 1}\right] \cdot V 2-\frac{1}{C 1 \cdot L 1} \cdot V 1+\frac{1}{R s \cdot C 1} \cdot f_{S}(\mathrm{t}) \forall f_{S}(\mathrm{t})=\dot{V}_{S}(\mathrm{t}) \\
& \frac{d V 3}{d t}=1 \quad \forall(\mathrm{V} 3(\mathrm{t})=\mathrm{t})
\end{aligned}
$$

First examine the case of $\lambda=0 \Rightarrow R s \cdot C 1 \rightarrow \infty, \mathrm{C} 1=$ const, then $\mathrm{Rs} \rightarrow \infty$

The limit cycle, the isolated periodic orbit, of the unforced oscillator of Van Der Pol becomes a cylinder; that is, topologically it is homeomorphism to $S^{1} x \mathbb{R}$. The cylinder is an invariant manifold in the sense that any solution starting on the cylinder remains on it for all positive time. This invariant cylinder attracts all nearby solutions. For $\lambda=0, \lambda \rightarrow 0, R s \rightarrow \infty$ the Active RFID TAG invariant cylinder is filled with a family of periodic solutions. The cylinder under the projection $\mathbb{R}^{2} x \mathbb{R} \rightarrow \mathbb{R}^{2}$ simply becomes the limit cycle. Actually Active RFID TAGs act as periodic forcing with small amplitude, that $|\lambda|$ small. In this case, there is still a cylinder in $\mathbb{R}^{2} x \mathbb{R}$ close to the invariant cylinder of the unforced oscillator. This new cylinder is an invariant manifold of solutions of the forced equation and attracts all nearby solutions. The flow on the invariant cylinder of the forced equation can be quite different from the one of the unforced oscillator. In Active RFID TAG concern to Van Der Pol's equation we get the equation: 


$$
\begin{aligned}
& \ddot{X}+\alpha \cdot \phi(x) \cdot \dot{X}+X=\lambda \cdot f_{S}(\mathrm{t}) \\
& \varepsilon>>1 \Rightarrow \ddot{V}+\left(\frac{1}{R 1}+\frac{1}{R s}\right) \cdot \frac{1}{C 1} \cdot \dot{V}+\frac{1}{L 1 \cdot C 1} \cdot V=\frac{1}{R s \cdot C 1} \cdot f_{S}(t) \\
& \varepsilon>1 \Rightarrow \ddot{V}+\left(\frac{1}{R 1}+\frac{1}{R s}\right) \cdot \frac{1}{C 1} \cdot \dot{V}+\frac{1}{L 1 \cdot C 1} \cdot V=\frac{1}{R s \cdot C 1} \cdot \dot{V}_{s}(t) \\
& \text { then } \phi(x)=1, \alpha=\left(\left(\frac{1}{R 1}+\frac{1}{R s}\right) \cdot \frac{1}{C 1}\right), \frac{1}{L 1 \cdot C 1} \rightarrow 1(L 1 \cdot C 1 \approx 1) \\
& \phi(x)=1>0 \quad \forall|\mathrm{t}|>1 \text { sec, } f_{S}(\mathrm{t}) \text { is T periodic and } \alpha, \beta \text { are non } \\
& \text { negative parameters. } \alpha=\left(\frac{1}{R 1}+\frac{1}{R s}\right) \cdot C 1, \beta=\frac{1}{R s \cdot C 1}
\end{aligned}
$$

Unforced investigation: $\lambda=0 \Rightarrow \frac{1}{R s} \rightarrow 0 \Rightarrow R s \rightarrow \infty$ then we return to Passive RFID TAG since the battery has a very high serial resistance - disconnected status.

\section{Active RFID TAG as a dynamic energy analysis}

Active RFID equivalent circuit total TAG power is a summation of all element's power.

$$
\begin{gathered}
P_{\text {total }}=\sum_{i=1}^{N} p_{i}=\text { TAG Power }, \sum_{i=1}^{N} p_{i}=\frac{1}{m} *\left[q(m) \cdot P_{T r x}+U(m) \cdot P_{T t x}+Z(m) \cdot P_{T r x}\right] \\
\sum_{i=1}^{N} p_{i}=p_{R s}+p_{C 1}+p_{R 1}+p_{L 1,}, \quad \text { energy } \Rightarrow W\left(t_{0}, t\right) \triangleq \int_{t 0}^{t} p\left(t^{\prime}\right) d t^{\prime}=\int_{t 0}^{t} v\left(t^{\prime}\right) \cdot i\left(t^{\prime}\right) d t^{\prime} \\
P(t)_{\text {total }}=\frac{d W\left(t_{0^{\prime}}, t\right)}{d t}=\frac{d}{d t}\left[\sum_{i=1}^{N} w_{i}\right], \quad \text { energy } \Rightarrow w_{\text {inductor }}=\frac{1}{2} \cdot L \cdot I^{2} \\
\text { energy } \Rightarrow w_{\text {capacitor }}=\frac{Q^{2}}{2 \cdot C}, P_{\text {resistor }}=I^{2} \cdot R, P_{R 1}=I_{R 1}^{2} \cdot R 1, P_{R s}=I_{R s}^{2} \cdot R s \\
\text { energy } \Rightarrow w_{L 1}=\frac{1}{2} \cdot L_{1} \cdot I_{L 1}^{2} \Rightarrow P_{L 1}=\frac{d}{d t} w_{L 1}=L \cdot I_{L 1} \cdot I_{L 1} \\
\text { energy } \Rightarrow w_{C 1}=\frac{Q_{C 1}^{2}}{2 \cdot C 1} \Rightarrow P_{C 1}=\frac{d}{d t} w_{C 1}=\frac{Q_{C 1} \cdot Q_{C 1}}{C 1} \\
\text { energy } \Rightarrow w w_{C 1}=\frac{C_{1} \cdot V_{C 1}^{2}}{2} \Rightarrow P_{C 1}=\frac{d}{d t} w_{C 1}=C_{1} \cdot V_{C 1} \cdot V_{C 1} \\
I_{L 1}=\frac{1}{L 1} \cdot \int_{0}^{t} V_{L 1} \cdot d t \Rightarrow I_{L 1}=\frac{V_{L 1}}{L 1}
\end{gathered}
$$




$$
\begin{gathered}
\sum_{i=1}^{N} p_{i}=I_{R 1}^{2} \cdot R 1+I_{R s}^{2} \cdot R s+L \cdot I_{L 1} \cdot \dot{I_{L 1}}+\frac{Q_{C 1} \cdot \dot{Q}_{C 1}}{C 1} \\
\sum_{i=1}^{N} p_{i}=\frac{V^{2}}{R 1}+\frac{[V-V s(t)]^{2}}{R s}+L \cdot I_{L 1} \cdot \dot{I_{L 1}}+C_{1} \cdot V_{C 1} \cdot \dot{V}_{C 1} \\
\sum_{i=1}^{N} p_{i}=V^{2} \cdot\left[\frac{1}{R 1}+\frac{1}{R s}\right]-\frac{2 \cdot V \cdot V s(t)}{R s}+\frac{[V s(t)]^{2}}{R s}+\frac{V}{L 1} \cdot \int_{0}^{t} V d t+C_{1} \cdot V \cdot \dot{V} \\
\frac{1}{m} *\left[q(m) \cdot P_{T r x}+U(m) \cdot P_{T t x}+Z(m) \cdot P_{T r x}\right]= \\
V^{2} \cdot\left[\frac{1}{R 1}+\frac{1}{R s}\right]-\frac{2 \cdot V \cdot V s(t)}{R s}+\frac{[V S(t)]^{2}}{R s}+\frac{V}{L 1} \cdot \int_{0}^{t} V d t+C_{1} \cdot V \cdot \dot{V}
\end{gathered}
$$

\section{Active RFID TAG fixed points and linearization}

$$
\begin{aligned}
& \frac{d V 1}{d t}=V 2 \\
& \frac{d V 2}{d t}=-\left[\frac{1}{C 1 \cdot R 1}+\frac{1}{R s \cdot C 1}\right] \cdot V 2-\frac{1}{C 1 \cdot L 1} \cdot V 1+\frac{1}{R s \cdot C 1} \cdot \dot{V}_{s}(\mathbf{t})
\end{aligned}
$$

Now we consider linear system: $\frac{d V 1}{d t}=f(V 1, V 2), \frac{d V 2}{d t}=g(V 1, V 2)$

And suppose that $\left(V_{1}^{*}, V_{2}^{*}\right)$ is a fixed point: $f\left(V_{1}^{*}, V_{2}^{*}\right)=0, \mathrm{~g}\left(V_{1}^{*}, V_{2}^{*}\right)=0$

Let $U 1=V 1-V_{1}^{*}, U 2=V 2-V_{2}^{*} \quad$ Denote the components of a small disturbance from the fixed point. To see whether the disturbance grows or decays, we need to derive differential equations for $\mathrm{U} 1$ and $\mathrm{U} 2$. Lets do the $\mathrm{U} 1$ equation first:

$$
\frac{d U 1}{d t}=\frac{d V 1}{d t} \text { since } V_{1}^{*} \text { is constant. }
$$

$\frac{d U 1}{d t}=\frac{d V 1}{d t}=f\left(U 1+V_{1}^{*}, \mathrm{U} 2+V_{2}^{*}\right)=f\left(V_{1}^{*}, V_{2}^{*}\right)+U 1 \cdot \frac{\partial f}{\partial V 1}+U 2 \cdot \frac{\partial f}{\partial V 2}+O\left(U_{1}^{2}, U_{2}^{2}, \mathrm{U} 1 \cdot \mathrm{U} 2\right)$ (Taylor series expansion)

To simplify the notation, we have written $\frac{\partial f}{\partial V 1}$ and $\frac{\partial f}{\partial V 2}$ these partial derivatives are to be evaluated at the fixed point $\left(V_{1}^{*}, V_{2}^{*}\right)$; thus they are numbers, not functions. Also the short hand notation $\mathrm{O}\left(\mathrm{U}_{1}^{2}, \mathrm{U}_{2}^{2}, \mathrm{U} 1 \cdot \mathrm{U} 2\right)$ denotes quadratic terms in U1 and U2. Since U1 and U2 are small, these quadratic terms are extremely small. Similarly we find $\frac{d U 2}{d t}=U 1 \cdot \frac{\partial g}{\partial V 1}+U 2 \cdot \frac{\partial g}{\partial V 2}+O\left(U_{1}^{2}, U_{2}^{2}, \mathrm{U} 1 \cdot \mathrm{U} 2\right)$, Hence the disturbance (U1, U2) evolves according to : 
The Matrix $\quad A=\left(\begin{array}{cc}\frac{\partial f}{\partial V 1} & \frac{\partial f}{\partial V 2} \\ \frac{\partial g}{\partial V 1} & \frac{\partial g}{\partial V 2}\end{array}\right)_{\left(V_{1}^{*}, V_{2}^{*}\right)}$ is called the Jacobian matrix at the fixed point $\left(V_{1}^{*}, V_{2}^{*}\right)$ and the Quadratic terms are tiny, its tempting to neglect them altogether.

If we do that, we obtain the linearized system. $\left(\begin{array}{l}\frac{d U 1}{d t} \\ \frac{d U 2}{d t}\end{array}\right)=\left(\begin{array}{cc}\frac{\partial f}{\partial V 1} & \frac{\partial f}{\partial V 2} \\ \frac{\partial g}{\partial V 1} & \frac{\partial g}{\partial V 2}\end{array}\right) \cdot\left(\begin{array}{l}U 1 \\ U 2\end{array}\right)$ whose dynamic can be analyzed by the general methods.

$$
\begin{aligned}
& f(V 1, V 2)=V 2 \\
& g(V 1, V 2)=-\left[\frac{1}{C 1 \cdot R 1}+\frac{1}{R s \cdot C 1}\right] \cdot V 2-\frac{1}{C 1 \cdot L 1} \cdot V 1+\frac{1}{R s \cdot C 1} \cdot \dot{V}_{S}(t) \\
& \frac{\partial f}{\partial V 1}=0, \frac{\partial f}{\partial V 2}=1, \frac{\partial g}{\partial V 1}=-\frac{1}{C 1 \cdot L 1}, \frac{\partial g}{\partial V 2}=-\left(\frac{1}{C 1 \cdot R 1}+\frac{1}{R s \cdot C 1}\right) \\
& \left(\begin{array}{l}
\frac{d U 1}{d t} \\
\frac{d U 2}{d t}
\end{array}\right)=\left(\begin{array}{cc}
0 & 1 \\
-\frac{1}{C 1 \cdot L 1} & \left.-\left[\frac{1}{C 1 \cdot R 1}+\frac{1}{R s \cdot C 1}\right]\right) \cdot\left(\begin{array}{l}
U 1 \\
U 2
\end{array}\right)
\end{array}\right.
\end{aligned}
$$

\section{Active RFID TAG stability analysis based on forced Van Der Pol's system}

The basic Active RFID Forced Van Der Pol's equation

$$
\begin{aligned}
& \varepsilon>1 \Rightarrow \ddot{V}+\left(\frac{1}{R 1}+\frac{1}{R s}\right) \cdot \frac{1}{C 1} \cdot \dot{V}+\frac{1}{L 1 \cdot C 1} \cdot V=\frac{1}{R s \cdot C 1} \cdot \dot{V}_{S}(t) \\
& \text { then } \phi(x)=1, \alpha=\left(\left(\frac{1}{R 1}+\frac{1}{R s}\right) \cdot \frac{1}{C 1}\right), \frac{1}{L 1 \cdot C 1} \rightarrow 1(L 1 \cdot C 1 \approx 1) \\
& \beta=\frac{1}{R s \cdot C 1}
\end{aligned}
$$

In our case $\phi(V)=1, \phi(V)>0$ for $|\mathrm{V}|>1$ and $\dot{V}_{S}(\mathrm{t})$ is $\mathrm{T}$ periodic and $\left(\frac{1}{R 1}+\frac{1}{R s}\right) \cdot \frac{1}{\mathrm{C} 1}, \frac{1}{R s \cdot \mathrm{C} 1}$ are non negative parameters. It is convenient to rewrite the Active RFID forced Van Der Pol's equation as an autonomous system. $\theta=\mathrm{t} \Rightarrow \frac{d \theta}{d t}=1$ 


$$
\begin{aligned}
& \dot{V}=\mathrm{Y}-\left(\frac{1}{R 1}+\frac{1}{R s}\right) \cdot \frac{1}{C 1} \cdot \phi(V) \\
& \dot{Y}=-\mathrm{V}+\frac{1}{R 1 \cdot C 1} \cdot \dot{V}_{S}(\theta) \\
& \dot{\theta}=1 \quad(\mathrm{~V}, \mathrm{Y}, \theta) \in \mathbb{R}^{2} x S^{1}
\end{aligned}
$$

$\phi(V)=1 \quad$ remain strictly positive as $|V| \rightarrow \infty$ for unforced system $\frac{1}{R 1 \cdot C 1} \cdot \dot{V}_{S}(\theta) \rightarrow 0$ but $\frac{1}{R 1 \cdot C 1} \neq 0$ then $\dot{V}_{S}(\theta)=0$ no energy is supply to the Active RFID TAG, become Passive RFID TAG. First we suppose that $\alpha \ll 1\left(\left(\frac{1}{R 1}+\frac{1}{R s}\right) \cdot \frac{1}{C 1} \ll 1\right)$ is a small parameter, so the autonomous system is a perturbation of linear oscillator. $\dot{V}=\mathrm{Y}, \dot{Y}=-\mathrm{V}$ which has a phase plane filled with circular periodic orbits each of period $2 \cdot \pi$. Using regular perturbation or averaging methods, we can show that precisely one of these orbits is preserved under the perturbation. Selecting the invertible transformation:

$$
\left(\begin{array}{l}
\xi 1 \\
\xi 2
\end{array}\right)=\left(\begin{array}{cc}
\cos (t) & -\sin (t) \\
-\sin (t) & -\cos (t)
\end{array}\right) \cdot\left(\begin{array}{l}
V \\
Y
\end{array}\right)
$$

which "freezes" the unperturbed system and the autonomous system become :

$$
\begin{aligned}
& \dot{\xi} 1=-\left(\frac{1}{R 1}+\frac{1}{R s}\right) \cdot \frac{1}{C 1} \cdot \cos t \cdot\left[(\xi 1 \cdot \cos (t)-\xi 2 \cdot \sin (t))^{3} / 3-(\xi 1 \cdot \cos (t)-\xi 2 \cdot \sin (t))\right] \\
& \dot{\xi} 2=-\left(\frac{1}{R 1}+\frac{1}{R s}\right) \cdot \frac{1}{C 1} \cdot \sin t \cdot\left[(\xi 1 \cdot \cos (t)-\xi 2 \cdot \sin (t))^{3} / 3-(\xi 1 \cdot \cos (t)-\xi 2 \cdot \sin (t))\right]
\end{aligned}
$$

this transformation is orientation reversing approximation the function $\xi 1, \xi 2$ which vary slowly because $\dot{\xi} 1, \dot{\xi} 2$ are small. Integrating each function with respect to time (t) from 0 to $\mathrm{T}=2 \cdot \pi$, holding $\xi 1, \xi 2$ fixed we obtain:

$$
\begin{aligned}
& \dot{\xi} 1=\left(\frac{1}{R 1}+\frac{1}{R s}\right) \cdot \frac{1}{C 1} \cdot \xi 1 \cdot\left[1-\left(\xi 1^{2}+\xi 2^{2}\right) / 4\right] / 2 \\
& \xi 2=\left(\frac{1}{R 1}+\frac{1}{R s}\right) \cdot \frac{1}{C 1} \cdot \xi 2 \cdot\left[1-\left(\xi 1^{2}+\xi 2^{2}\right) / 4\right] / 2
\end{aligned}
$$
this system is correct at first order, but there is an error of $\mathrm{O}\left(\left[\left(\frac{1}{R 1}+\frac{1}{R_{S}}\right) \cdot \frac{1}{C 1}\right]\right)$. In polar
coordinates, we therefore have

$$
\begin{aligned}
& \dot{r}=\frac{r}{2} \cdot\left(\frac{1}{R 1}+\frac{1}{R s}\right) \cdot \frac{1}{C 1} \cdot\left(1-\frac{r^{2}}{4}\right)+O\left(\left[\left(\frac{1}{R 1}+\frac{1}{R s}\right) \cdot \frac{1}{C 1}\right]^{2}\right) \\
& \dot{\varphi}=0+O\left(\left[\left(\frac{1}{R 1}+\frac{1}{R s}\right) \cdot \frac{1}{C 1}\right]^{2}\right)
\end{aligned}
$$


Neglecting the $\mathrm{O}\left(\left[\left(\frac{1}{R 1}+\frac{1}{R s}\right) \cdot \frac{1}{C 1}\right]^{2}\right)$ terms this system has an attracting circle of fixed points at $\mathrm{r}=2$ reflecting the existence of a one parameter family of almost sinusoidal solutions: $\quad \mathrm{V}=\mathrm{r}(\mathrm{t}) \cdot \cos (\mathrm{t}+\varphi(t)) \quad$ with slowly varying amplitude $\mathrm{r}(\mathrm{t})=2+\mathrm{O}\left(\left[\left(\frac{1}{R 1}+\frac{1}{R s}\right) \cdot \frac{1}{C 1}\right]^{2}\right)$ and the phase $\varphi(t)=\varphi^{0}+\mathrm{O}\left(\left[\left(\frac{1}{R 1}+\frac{1}{R s}\right) \cdot \frac{1}{C 1}\right]^{2}\right)$

Constant $\varphi^{0}$ being determined by initial conditions. When the value of $\left(\frac{1}{R 1}+\frac{1}{R S}\right) \cdot \frac{1}{C 1}$

Is not small the averaging procedure no longer works and other methods must be used.

The investigation can be done for Active RFID's system forced Van Der Pole. Lets consider $\dot{V}_{S}(\mathrm{t}) \neq 0$ we suppose $\left(\frac{1}{R 1}+\frac{1}{R s}\right) \cdot \frac{1}{C 1}, \frac{1}{R s \cdot C 1} \ll 1$ and use the same transformation as we use in the unforced system $\dot{V}_{S}(\mathrm{t})=0$. when we interest in the periodic forced response we use the $\frac{2 \cdot \pi}{\omega}$ periodic transformation.

$$
\begin{aligned}
& \left(\begin{array}{l}
\xi 1 \\
\xi 2
\end{array}\right)=\left(\begin{array}{cc}
\cos (\omega t) & -\frac{1}{\omega} \cdot \sin (\omega t) \\
-\sin (\omega t) & -\frac{1}{\omega} \cdot \cos (\omega t)
\end{array}\right) \cdot\left(\begin{array}{l}
V \\
Y
\end{array}\right) \\
& \dot{\xi} 1=-\left(\frac{1}{R 1}+\frac{1}{R s}\right) \cdot \frac{1}{C 1} \cdot \phi(V) \cdot \cos (\omega \cdot t)-\left(\frac{\omega^{2}-1}{\omega}\right) \cdot V \cdot \sin (\omega \cdot t)-\frac{1}{R s \cdot C 1 \cdot \omega} \cdot \sin \left(\omega \cdot t \cdot \dot{V}_{S}(t)\right) \\
& \dot{\xi} 2=\left(\frac{1}{R 1}+\frac{1}{R s}\right) \cdot \frac{1}{C 1} \cdot \phi(V) \cdot \sin (\omega \cdot t)-\left(\frac{\omega^{2}-1}{\omega}\right) \cdot V \cdot \cos (\omega \cdot t)-\frac{1}{R s \cdot C 1 \cdot \omega} \cdot \cos \left(\omega \cdot t \cdot \dot{V}_{S}(\mathrm{t})\right) \\
& \frac{1}{C 1 \cdot L 1} \rightarrow 1, \phi(V)=1 \text { in our case. } \\
& \dot{\xi} 1=-\left(\frac{1}{R 1}+\frac{1}{R s}\right) \cdot \frac{1}{C 1} \cdot \cos (\omega \cdot t)-\left(\frac{\omega^{2}-1}{\omega}\right) \cdot V \cdot \sin (\omega \cdot t)-\frac{1}{R s \cdot C 1 \cdot \omega} \cdot \sin \left(\omega \cdot t \cdot \dot{V}_{S}(t)\right) \\
& \dot{\xi} 2=\left(\frac{1}{R 1}+\frac{1}{R s}\right) \cdot \frac{1}{C 1} \cdot \sin (\omega \cdot t)-\left(\frac{\omega^{2}-1}{\omega}\right) \cdot V \cdot \cos (\omega \cdot t)-\frac{1}{R s \cdot C 1 \cdot \omega} \cdot \cos \left(\omega \cdot t \cdot \dot{V}_{S}(\mathrm{t})\right)
\end{aligned}
$$

\section{Summery}

Active RFID TAG system can be represent as Voltage source (internal resistance), Parallel Resistor, Capacitor, and Inductance circuit. Linear bifurcation system explain Active RFID TAG system behavior for any initial condition $V(t)$ and $d V(t) / d t$. Active RFID's Coil is a very critical element in Active RFID TAG functionality. Optimization can be achieved by Coil's parameters inspection and System bifurcation controlled by them. Spiral, Circles, and other Active RFID phase system behaviors can be optimize for better Active RFID TAG 
performance and actual functionality. Active RFID TAG losses also controlled for best performance and maximum efficiency.

\section{References}

[1] Yuri A. Kuznetsov, Elelments of Applied Bifurcation Theory. Applied Mathematical Sciences.

[2] Jack K. Hale. Dynamics and Bifurcations. Texts in Applied Mathematics, Vol. 3

[3] Steven H. Strogatz, Nonlinear Dynamics and Chaos. Westview press

[4] John Guckenheimer, Nonlinear Oscillations, Dynamical Systems, and Bifurcations of Vector Fields. Applied Mathematical Sciences Vol 42.

[5] Stephen Wiggins, Introduction to Applied Nonlinear Dynamical Systems and Chaos. Text in Applied Mathematics (Hardcover).

[6] O. Aluf "RFID TAGs COIL's Dimensional Parameters Optimization As Excitable Linear Bifurcation Systems" (IEEE COMCAS2008 Conference, May 2008). 


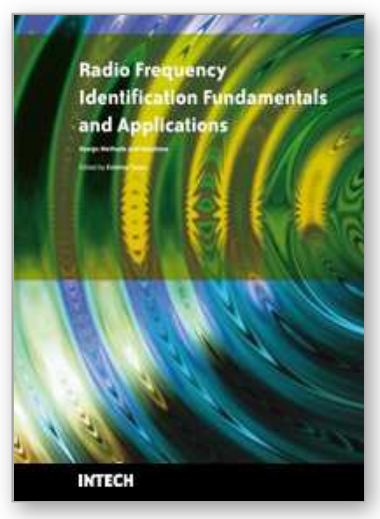

\author{
Radio Frequency Identification Fundamentals and Applications \\ Design Methods and Solutions \\ Edited by Cristina Turcu
}

ISBN 978-953-7619-72-5

Hard cover, 324 pages

Publisher InTech

Published online 01, February, 2010

Published in print edition February, 2010

This book, entitled Radio Frequency Identification Fundamentals and Applications, Bringing Research to Practice, bridges the gap between theory and practice and brings together a variety of research results and practical solutions in the field of RFID. The book is a rich collection of articles written by people from all over the world: teachers, researchers, engineers, and technical people with strong background in the RFID area. Developed as a source of information on RFID technology, the book addresses a wide audience including designers for RFID systems, researchers, students and anyone who would like to learn about this field. At this point I would like to express my thanks to all scientists who were kind enough to contribute to the success of this project by presenting numerous technical studies and research results. However, we couldn't have published this book without the effort of InTech team. I wish to extend my most sincere gratitude to InTech publishing house for continuing to publish new, interesting and valuable books for all of us.

\title{
How to reference
}

In order to correctly reference this scholarly work, feel free to copy and paste the following:

Ofer Aluf (2010). Active RFID TAGs System Analysis of Energy Consumption As Excitable Linear Bifurcation System, Radio Frequency Identification Fundamentals and Applications Design Methods and Solutions, Cristina Turcu (Ed.), ISBN: 978-953-7619-72-5, InTech, Available from:

http://www.intechopen.com/books/radio-frequency-identification-fundamentals-and-applications-designmethods-and-solutions/active-rfid-tags-system-analysis-of-energy-consumption-as-excitable-linear-bifurcationsystem

\section{INTECH}

open science | open minds

\author{
InTech Europe \\ University Campus STeP Ri \\ Slavka Krautzeka 83/A \\ 51000 Rijeka, Croatia \\ Phone: +385 (51) 770447 \\ Fax: +385 (51) 686166 \\ www.intechopen.com
}

\author{
InTech China \\ Unit 405, Office Block, Hotel Equatorial Shanghai \\ No.65, Yan An Road (West), Shanghai, 200040, China \\ 中国上海市延安西路65号上海国际贵都大饭店办公楼405单元 \\ Phone: $+86-21-62489820$ \\ Fax: $+86-21-62489821$
}


(C) 2010 The Author(s). Licensee IntechOpen. This chapter is distributed under the terms of the Creative Commons Attribution-NonCommercialShareAlike-3.0 License, which permits use, distribution and reproduction for non-commercial purposes, provided the original is properly cited and derivative works building on this content are distributed under the same license. 